
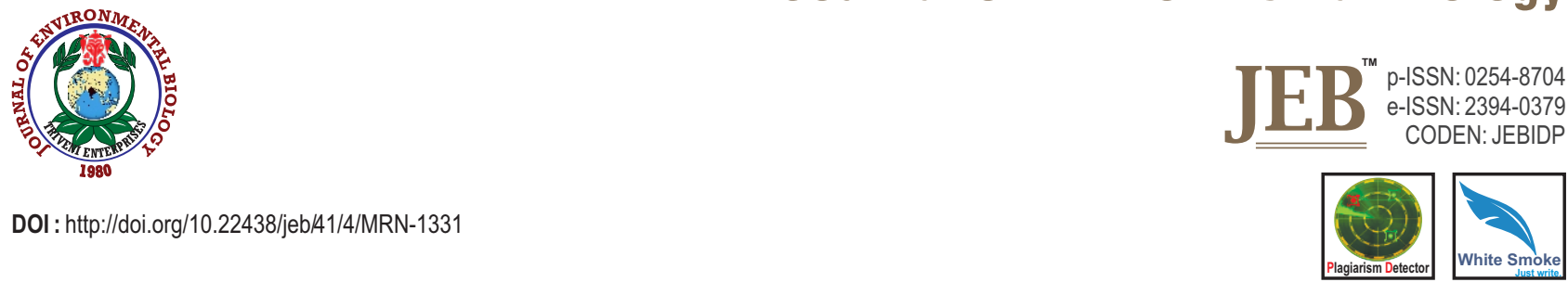

\title{
Analysis of bacterial diversity associated with polyketide synthase (PKS) gene in brackish metagenome of TsoKar Lake of Ladakh, India
}

\author{
V.K. Gupta, S.S. Khan, V. Verma and S. Rasool* \\ School of Biotechnology, Shri Mata Vaishno Devi University, Katra - 182 320, India \\ *Corresponding Author Email : shafaq.rasool@smvdu.ac.in
}

\section{Abstract}

Aim: To explore bacterial diversity associated to polyketide synthase (PKS) genes in the TsoKar brackish metagenome.

Methodology: The metagenomic DNA of TsoKar water and sediment samples were isolated and amplified using PKS specific degenerate primers. PCR amplified products were analysed using lon Torrent PGM platform and revealed a total of 2,11,030 reads with 534 contigs and $55 \%$ of $G+C$ content. The results of BLAST $x$ was further analyzed for species diversity using MEGAN5 software.

Results: Halomonas was found to be the dominant phyla followed by Cyanobium, Acinetobacter and Burkholderia, respectively, along with majority of uncultured microorganisms present in TsoKar metagenome. Since TsoKar is a brackish water lake, and brackish aquatic ecosystems are a major hub of diverse microbes was also revealed in this study. TsoKar metagenomic sequence with accession number SRR8381943 was deposited in SRAdatabase.

Interpretation: TsoKar Lake is considered as one of the most important biosphere reserves. The results of this study confirm that this unique niche harbors good bacterial diversity associated with polyketide synthase genes, which justifies the idea of bioprospecting such niches for the discovery of novel metabolites like antimicrobial compounds using next-generation sequencing approach.

Keywords: Brackish water, Diversity, Metagenome, NGS technology, PKS gene

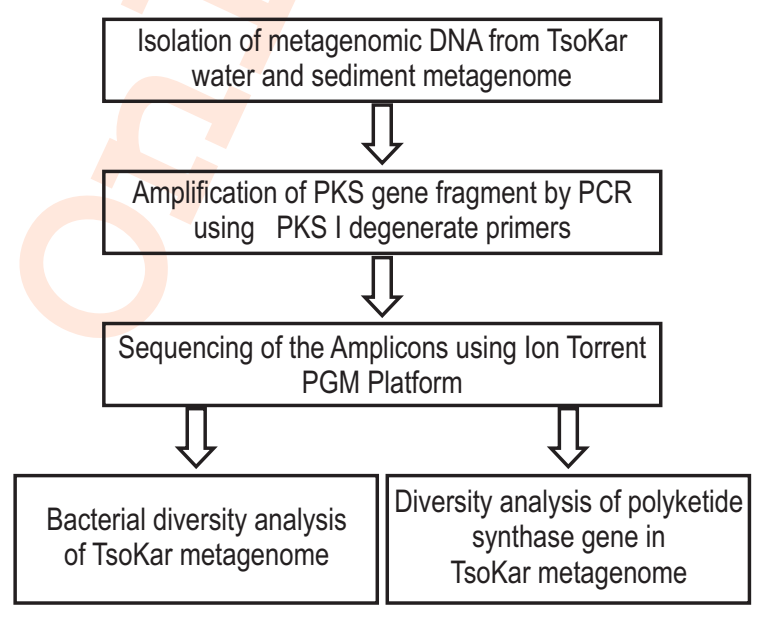

How to cite : Gupta, V.K., S.S. Khan, V. Verma and S. Rasool: Analysis of bacterial diversity associated with polyketide synthase (PKS) gene in brackish metagenome of TsoKar Lake of Ladakh, India. J. Environ. Biol., 41, 755-760 (2020). 


\section{Introduction}

Microorganisms are the largest reservoir of potentially valuable natural compounds, such as nonribosomal peptides, alkaloids and polyketides. (Zhao et al., 2011). Microorganism culturability in extreme environments is typically less, creating the need to explore culture-independent techniques to determine the microbes functional and taxonomic status (Mangrola et al., 2015). These culture-independent techniques have been used successfully to analyze microbial communities in brackish water metagenome. Metagenome is the collective genome of microorganisms from an environmental sample to provide access, and classify, this untapped diversity of microbes and discover new genes, metabolic pathways, and essential products relevant to different fields like pharmaceutical, biotechnology etc. (Handelsman, 2004).

Polyketides are one of the largest groups of natural products with enormous diversity of structures and complex chemical architectures. Polyketide biosynthetic pathways are usually divided into three main groups: Type I, Type II and Type III. Polyketides (PKs) are the most significant secondary metabolites such as anticancer drugs (epothilone), antifungals (amphotericin B), insecticides (spinosyn A) and antibiotics (erythromycin) which contributes in medicine, agriculture and industry (Barajas et al., 2017). Multimodular enzyme complexes are known as Type I modular polyketide synthases (PKS-I) biosynthesize these particular examples of polyketides. Type II PKSs consist of individual proteins conducting iteratively one enzymatic activity to catalyze the production of aromatic polyketides such as antibiotic actinorhodin (Hertweck, 2009; Zhang et al., 2017). Type III PKSs are involved in small aromatic compounds assembly and are present in plants and bacteria (Katz and Baltz, 2016; Moore and Hopke, 2001).

The highly modular design of PKS biosynthetic system offers a basis for discovering a wide range of gene clusters resulting in a variety of chemical repertoires, including several clinically useful microbial metabolites (Cragg and Newman, 2013). In the present scenario, the discovery of new polyketides is one of the promising leads for discovering therapeutically important drugs.

Primers specific to the conservative regions of KSdomain of PKS are successfully used for detection and identification of genes responsible for the synthesis of secondary metabolites of polyketide nature in the bacterial genomes (Palomo et al., 2013; Sukhanova et al., 2017; Wu et al., 2011). The sequences of PKS modules can be analyzed by PCR corresponding to gene clusters in microbial communities. PKS gene clusters from unculturable microbes have been identified taking part in the synthesis of known or unknown polyketides from different metagenomic libraries like soil (Courtois et al., 2003; Parsley et al., 2011), beetles (Piel, 2002), sponges (Kaluzhnaya et al., 2012; Kurnia etal., 2017) etc.
Brackish water lakes are the bacterial community's most exceptional reservoir with tolerance adaptability to saline stress. The metagenomic approach has been applied in the present study to gain insight into the polyketide synthase gene diversity associated with TsoKar metagenome. Due to low cost of DNA sequencing and advances in bioinformatics, sequencing approach in metagenomics has become increasingly popular in recent decades. High-throughput next-generation sequencing has emerged as a valuable technique for investigating more thoroughly the role of polyketide synthase gene diversity in microbial ecology to access the vast uncultured microbial complex diversity present in the environment (Ziemert et al., 2016). In the present study, a set of degenerate oligonucleotide primers was used for amplifying PKS gene fragments from TsoKar metagenome, a high altitude brackish water lake situated $4460 \mathrm{~m}$ above mean sea level in the North-western Himalayas of Ladakh region, India. TsoKar is also referred to as "White Lake" due to deposition of white salt found all over the shores of the lake. The amplified PKS gene fragments were sequenced through NGS Ion Torrent Technology to explore the diversity of polyketide synthase genes present in the TsoKar metagenome. Metagenomic analysis using high throughput screening can serve as a valuable tool for exploring bacterial diversity associated with polyketide synthase gene diversity in brackish water metagenomes like TsoKar lake. Polyketide synthase genes are involved with the production of secondary metabolites of huge importance having antibiotic and anticancer activity.

The aim of the current study was to understand bacterial diversity associated with PKS gene diversity in extreme environments such as brackish lake of TsoKar water sample. The study focused on the identification of community correlations to elucidate the bacterial diversity associated with PKS biosynthetic genes to provide insights about the potential of TsoKar metagenome for the production of polyketide bioactive molecules.

To the best of our knowledge, this is the first study to explore bacterial diversity of PKS genes in brackish metagenome of TsoKar lake of Ladakh region, India.

\section{Materials and Methods}

Sample collection and DNA isolation : Water and sediment samples from TsoKar lake ( $33^{\circ} 18^{\prime} \mathrm{N}$ and $\left.77^{\circ} 59^{\prime} \mathrm{E}\right)$ situated 4460 $\mathrm{m}$ above sea level were collected from various sites from the depth ranging from 5 to $20 \mathrm{~cm}$ in sterile containers. The $\mathrm{pH}$ of lake was 8.8 and temperature of $25^{\circ} \mathrm{C}$. The samples were collected in the month of October 2015 and stored at $4^{\circ} \mathrm{C}$. Isolation of metagenomic DNA from these samples was performed in the Molecular Biology Laboratory at School of Biotechnology, Shri Mata Vaishno Devi University, Katra, J\&K within two weeks from the date of sample collection. 
Metagenomic DNA extraction from water and sediment sample was done using "Meta-G-Nome ${ }^{\mathrm{TM}}$ DNA Isolation Kit" (epicentre, USA) and DNEASY Power soil Kit (Qiagen) following manufacturer's guidelines. High purity DNA isolated was further used in downstream applications. The purity and yield of metagenomic DNA isolated from water and sediment samples was measured at $230 \mathrm{~nm}, 260 \mathrm{~nm}$ and $280 \mathrm{~nm}$ using UV spectrophotometer (UV-1800, Shimadzu). Qualitative analysis of metagenomic DNA was done using absorbance ratio at A260 $\mathrm{nm} / 230 \mathrm{~nm}$ (DNA/ humic acid) and A260 nm/280 nm (DNA/Protein). Isolated DNA was checked on $0.8 \%$ agarose gel and stained with ethidium bromide to determine the size and concentration. All isolated DNAs were stored at $-20^{\circ} \mathrm{C}$ until further use.

Amplification of polyketide synthase gene fragment by PCR: Polyketide synthase gene fragment was amplified from water and sediment metagenomic DNA by PCR using one set of degenerate primers targeting highly conserved regions of type I PKS (PKS I) genes. Amplification for PKS type I was carried out by using one set of degenerate primer as PKSI primer set: sense, 5'CCSCAGSAGCGCSTSCTSCTSGA-3' and antisense 5'GTSCCSGTSCCGTGSGCCTCSA-3' (Courtois et al., 2003) with a Mastercycler Gradient (Eppendorf). The primer set was designed from polyketide producing actinomycetes which is complementary to highly conserved region of type I PKS genes. Specificity of this primer set confirmed that they specifically amplified the region of ketoacyl synthetase domain of polyketide producing strains like Streptomyces ambofaciens, Streptomyces coelicolor, Streptomyces rimosus and Streptomyces lactamandurans, and non-homologous strains like Bacillus licheniformis and Bacillus subtilis. Amplification reaction was performed by using the initial denaturation step at $94^{\circ} \mathrm{C}$ for $10 \mathrm{~min}$ followed by 30 cycles of DNA denaturation at $94^{\circ} \mathrm{C}$ for $30 \mathrm{sec}$., primer annealing at $55^{\circ} \mathrm{C}$ for $1 \mathrm{~min}$ and DNA strand extension at $72^{\circ} \mathrm{C}$ for $1 \mathrm{~min}$ and a final extension step at $72^{\circ} \mathrm{C}$ for $10 \mathrm{~min}$. PCR products were analyzed on $1 \%$ agarose gel.

Sequence analysis of TsoKar metagenome: The amplified products of TsoKar water and sediment samples generated with PKSI primer set was mixed and submitted to M/s. Bioserve Biotechnologies India Pvt. Ltd., Hyderabad, India for sequence analysis using NGS technology lon Torrent Platform. Raw data was processed to obtain high-quality clean reads using Trimmomatic version 0.35 . Filtered high-quality reads of the sample were assembled into scaffolds using CLC Genomics Work version 9.0. The result of BLASTx was further analyzed for species diversity using MEGAN5 software. MEGAN (Metagenome Analyzer) allows analysis of large data sets. In a pre-processing step, the set of DNA reads (or contigs) was compared against databases of known sequences using comparison tool such as BLAST. MEGAN was then used to estimate and interactively explore the taxonomical content of data set using NCBI taxonomy to summarize and order the results. The parameters used for analysis were Cutoff quality score for quality control (FastQC Tool Kit): >=20, Cutoff read length for high quality reads (FastQC Tool Kit): 70\%, Sequence identity threshold (CDHIT): 0.99 or $99 \%$, Database for RAPSearch: NCBI NR, E value (RAPSearch) : $<=0.001$ or $\log 10$ (e value) $<=-3$, Percent identity (RAPsearch): $>=30$.

\section{Results and Discussion}

The concentration and size of isolated DNA was validated by comparing it with Fosmid Control DNA (40 Kb; $\left.100 \mathrm{ng} \mathrm{\mu l}^{-1}\right)$ via gel electrophoresis on $0.8 \%$ agarose gel. Agarose gel was observed under UV light. The isolation of metagenomic DNA from TsoKar water and sediment sample is shown in Fig.1(a). The yield and purity of extracted metagenomic DNA from TsoKar water and sediment samples were analyzed by double-beam UV-VIS spectrophotometer (Shimadzu, UV-1800). Spectrophotometric analysis of the samples confirmed the purity of samples with respect to protein, RNAand humic acid contamination.

For studying the diversity of bacteria associated with polyketide synthase gene fragments in TsoKar metagenome, the metagenomic DNA of water and sediment samples was amplified
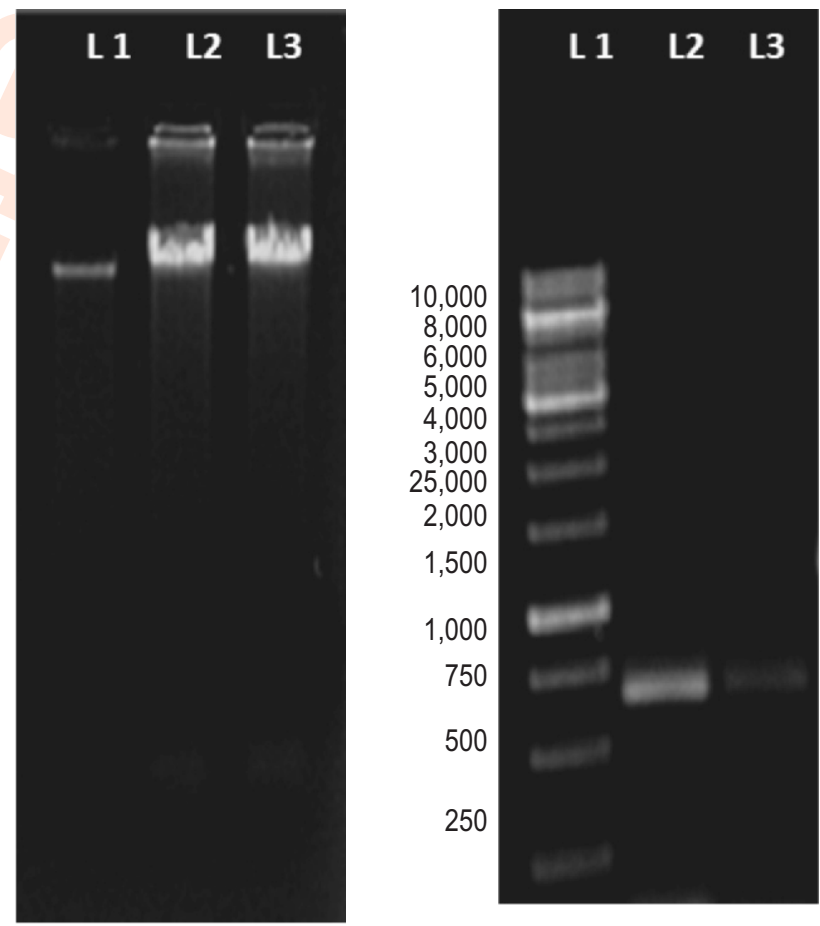

Fig. 1 (a) : $0.8 \%$ Agarose gel showing Metagenomic DNA isolated from TsoKar water and sediment Sample; Lane 1- Fosmid Control DNA, Lane 2- Metagenomic DNA from water sample, Lane 3- Metagenomic DNA from sediment sample; (b) Amplification of polyketide synthase gene fragment from TsoKar water and sediment sample; Lane1- $1 \mathrm{~Kb}$ DNA Ladder, Lanes 2 and 3-PCR amplified product (700bp) of water and 
with degenerate primers specific for PKS I gene fragment. The size of the amplified products was 700 bp as shown in Fig.1(b). The amplified products of water and sediment samples were purified and mixed for analysis by lon Torrent PGM Technology.

After De novo assembly, the total reads obtained were $2,11,030$ bp with a $\mathrm{G}+\mathrm{C}$ content of $55 \%$ and average base quality (Phred score) of 30.534 contigs were generated and subjected to BLASTx against nrDB database using Rap Search2 tool to assess the enrichment of polyketide synthase gene in TsoKar metagenome. The sequence length of TsoKar metagenome ranged from 50 to $255 \mathrm{bp}$.

In order to find the polyketide synthase gene enrichment in the sequenced sample, the species diversity was determined. The sample was found to be enriched with Halomonas sp. as shown in Fig. 2 and the presence of a large number of bacterial species belonging to bacterial phyla like Chromohalobacter, Halomonadaceae, Oceanospirilales and unculturable bacteria.

Diversity analysis of PKS gene fragment of TsoKar metagenome as shown in Fig. 3 , indicated a good diversity in PKS gene fragment, and it was found that PKS gene fragment was present in different species such as Cyanobium, Halomonas,

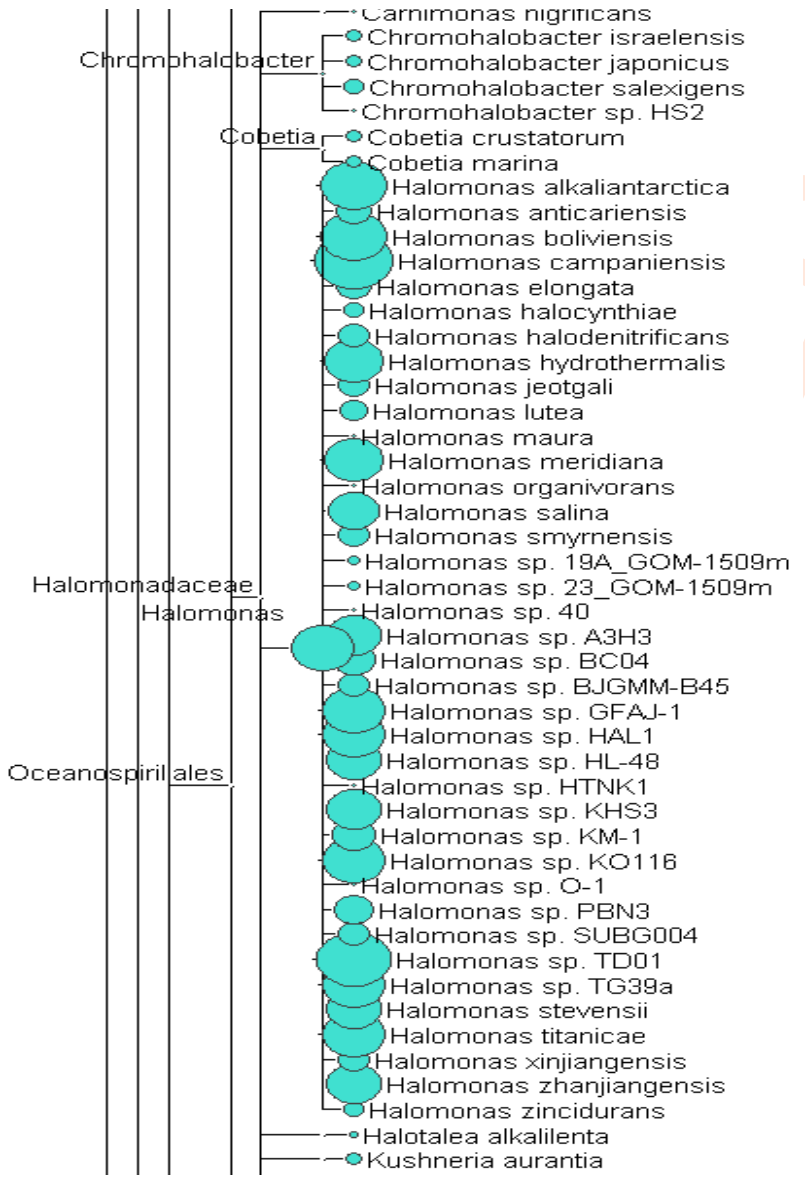

Fig. 2 : Analysis of bacterial diversity of TsoKar metagenome.
Acinetobacter, Burkholderia etc. PKS gene diversity from Halomonas, Acinetobacter, Burkholderia is less explored so bioprospecting this unique niche of TsoKar sample could provide some novel leads from these microorganisms for the discovery of drug molecules. It was also analysed that a good portion of PKS gene diversity in TsoKar metagenome was contributed by uncultivable bacteria as well which justifies the use of metagenomics in exploring the potential of brackish water metagenomes for PKS genes, which in turn can prove to be a useful niche for exploring novel bioactive molecules.

In this study, aim was to explore the diversity of sequences assigned to PKS genes in the brackish metagenome of TsoKar lake, allowing greater insights into potentially novel synthetic pathways and biocatalysts. Certain studies have already shown that environments are endowed with metabolic novelty as a useful precursor to metagenomic biosynthetic gene cluster mining for PKS genes (polyketides synthase) which can provide leads for new antimicrobial metabolites. PKS gene diversity has been reported in the Yellow Sea sediment and Actinobacteria was seen as the typical PKs and NRPs producers accounting for $0.82 \%$ of all the bacterial species of the sediment sample (Wei et al., 2018) contrary to our results. We were not able to identify PKS gene diversity accounted by Actinobacterial species, however, a major contributor of PKS gene diversity in our study is Halomonas species which could be due to the presence of the brackish environment.

PKS gene diversity reported from microbial community structure associated with fresh water sponge, Lubomirskia baicalensis identified ketosynthase domains in various types of microorganisms belonging to alpha-, beta-, delta-Proteobacteria, Verrucomicrobia, Cyanobacteria, Chlorophyta (Kaluzhnaya et al., 2012) similar to our results. It has also been shown that the microbiome of marine sponge Arenosclera brasiliensis harbors a large diversity of type I PKS subtypes belonging to Actinobacteria, Cyanobacteria, Alphaproteobacteria and Deltaproteobacteria (Burkholderia) (Trindade-Silva et al., 2012) as reported in our study, besides the presence of Halomonas in our sample.

PKS and NRPS gene diversities have also been studied in Antartic sediment samples showing the identification of Ketosyntase domains from diverse bacterial groups belonging to Proteobacteria, Firmicutes, Planctomycetes, Cyanobacteria, Actinobacteria, and some uncultured symbiotic bacteria (Zhao et al., 2008). Their results revealed great diversity and novelty of both PKS and NRPS genes in Antartic sediment.

Since it is already established that polyketides are a large class of compounds with interesting and valuable functions that are structurally complex. Such compounds are widely used for clinical applications such as, antifungals, antibiotics and narcotics. Polyketides can be typically derived from biological 


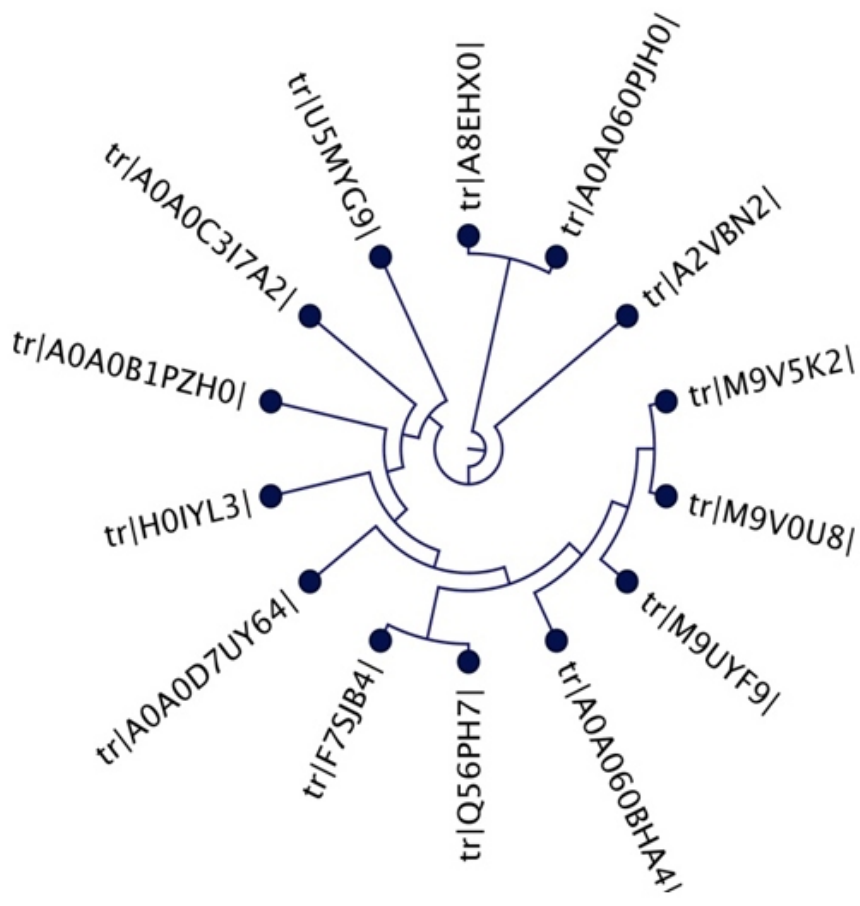

tr|A2VBN2|: Uncultured bacterium

tr|M9V0U8|: Cyanobium sp. LEGE 07186

tr|M9V5K2|: Cyanobium sp. LEGE 06134

tr|M9UYF9|: Cyanobium sp. LEGE 06012

$\operatorname{tr|} \mid \mathrm{A0A060BHA4|:} \mathrm{Halomons} \mathrm{campaniensis}$

$\operatorname{tr} \mid \mathrm{Q} 56 \mathrm{PH} 7 \mathrm{|}$ : Halomonas sp.

tr|F7SJB4|: Halomonas sp. TD01

tr|A0A0D7UY64|: Halomonas meridiana

tr|HOIYL3|: Halomonas sp. GFAJ-1

$\operatorname{tr}|\mathrm{AOA0B} 1 \mathrm{PZH} 0|$ : Halomonas hydrothermalis

$\operatorname{tr|}|\mathrm{A0A0C} 317 \mathrm{~A} 2|$ : Halomonas sp.

tr|U5MYG9|: Acinetobacter sp.

$\operatorname{tr|} \mid \mathrm{A} 8 \mathrm{EXHO}$ |: Burkholderia pseudomallei

$\operatorname{tr|} \mid$ A0A060PHJO|: Burkholderia sp.

Fig. 3 : Analysis of bacterial diversity associated with polyketide synthase genes in TsoKar metagenome.

sources or synthesized chemically (Musiol-Kroll and Wohlleben, 2018). With this background in mind, this study could provide as a basis for exploring the brackish niche of TsoKar lake for mining of novel bioactive molecules using culture dependent and culture independent approaches. The results of this study demonstrate the applicability of sequence-based approaches in gaining information about PKS gene diversity in brackish metagenomes for gaining valuable insights for further study.

The methodology developed in this study will assist future studies for obtaining new PKS genes, to search new biosynthetic pathways in the brackish metagenome. Metagenome sequence analysis can significantly provide an important breakthrough in depicting the taxonomic structure of brackish lakes like TsoKar, promising novel microbes and novel genes for biotechnological applications.

Accession number : The nucleotide sequence was submitted to the NCBI Sequence Read Archive with accession number SRR8381943.

\section{Acknowledgment}

This work was supported by the Council of Scientific and Industrial Research (CSIR), New Delhi, Government of India under grant number 37(1545)/12/EMR-II entitled "Exploring microbial diversity and mining novel hydrolases from brackish water lakes of Ladakh region by metagenomic approach".

\section{References}

Barajas, J.F., J.M. Blake-Hedges, C.B. Bailey, S. Curran and J.D. Keasling: Engineered polyketides: Synergy between protein and host level engineering. Synth. Syst. Biotechnol., 2, 147-166 (2017).

Cragg, G.M. and D.J. Newman: Natural products: A continuing source of novel drug leads. Biochimica et Biophysica Acta (BBA)-General Subjects, 1830, 3670-3695 (2013).

Courtois, S., C.M. Cappellano, M. Ball, F.X. Francou, P. Normand, G. Helynck, A. Martinez, S.J. Kolvek, J. Hopke and M.S. Osburne: Recombinant environmental libraries provide access to microbial diversity for drug discovery from natural products. Appl. Environ. Microbiol., 69, 49-55 (2003).

Handelsman, J.: Metagenomics: Application of genomics to uncultured microorganisms. Microbiol. Mol. Biol. Rev., 68, 669-685(2004).

Hertweck, C.: The biosynthetic logic of polyketide diversity. Angewandte Chemie International Edition, 48, 4688-4716 (2009).

Katz, L. and R.H. Baltz: Natural product discovery: Past, present and future. J. Ind. Microbiol. Biotechnol., 43, 155-176 (2016).

Kaluzhnaya, O.V., N.V. Kulakova and V.B. Itskovich: Diversity of Polyketide Synthase (PKS) genes in metagenomic community of freshwater sponge Lubomirskia baicalensis. Mol. Biol., 46, 790$795(2012)$

Kurnia, N.M., A.R. Uria, Y. Kusnadi, L. Dinawati, D.S. Zilda, T.A. Hadi, S. Setyahadi and F. Felix: Metagenomic survey of potential symbiotic bacteria and polyketide synthase genes in an Indonesian marine sponge. HAYATI J. Biosci., 24, 6-15 (2017).

Mangrola, A., P. Dudhagara, P. Koringa, C. Joshi, M. Parmar and R. Patel: Deciphering the microbiota of Tuwa hot spring, India using 
shotgun metagenomic sequencing approach. Genomics Data, 4, 153-155 (2015).

Moore, B.S. and J.N. Hopke: Discovery of a new bacterial polyketide biosynthetic pathway. Chembiochem., 2, 35-38 (2001).

Musiol-Kroll, E.M. and W. Wohlleben: Acyltransferases as tools for polyketide synthase engineering. Antibiotics, 7, 1-35 (2018).

Palomo S., I. Gonzalez, M. de la Cruz, J. Martín, J. Tormo, M. Anderson, R. Hill, F. Vicente, F. Reyes and O. Genilloud: Sponge-derived Kocuria and Micrococcus spp. as sources of the new thiazolyl peptide antibiotic kocurin. Mar. Drugs, 11, 1071-1086 (2013).

Parsley, L.C., J. Linneman, A.M. Goode, K. Becklund, I. George, R.M. Goodman, N.B. Lopanik and M.R. Liles: Polyketide synthase pathways identified from a metagenomic library are derived from soil acidobacteria. FEMS Microbiol. Ecol., 78, 176-187 (2011).

Piel, J.: A polyketide synthase-peptide synthetase gene cluster from an uncultured bacterial symbiont of Paederus beetles. PNAS, 99, 14002-14007 (2002).

Sukhanova, E., E. Zimens, V. Parfenova and O. Belykh: Diversity of polyketide synthase genes in the genomes of heterotrophic microorganisms isolated from epilithic biofilms of lake Baikal. Moscow Univ. Biol.Sci. Bull., 72, 211-217 (2017).

Trindade-Silva, A.E., C. Rua, G.G. Silva, B.E. Dutilh, A.P.B. Moreira, R.A.
Edwards, E. Hajdu, G. Lobo-Hajdu, A.T. Vasconcelos, R.G. Berlinck and F.L. Thompson: Taxonomic and functional microbial signatures of the endemic marine sponge Arenosclera brasiliensis. PLOS ONE, 7, 1-10 (2012).

Wei, Y., L.. Zhang, Z. Zhou and X. Yan: Diversity of gene clusters for polyketide and nonribosomal peptide biosynthesis revealed by metagenomic analysis of the yellow sea sediment. Front. Microbiol., 9, 295 (2018).

Wu, X.C., C.D. Qian, H.H. Fang, Y.P. Wen, J.Y. Zhou, Z.J. Zhan, R. Ding, $\mathrm{O}$. Li and H. Gao: Paenimacrolidin, a novel macrolide antibiotic from Paenibacillus sp. F6 B70 active against methicillin resistant Staphylococcus aureus. Microb. Biotechnol., 4, 491-502 (2011).

Zhao, J., N. Yang and R. Zeng: Phylogenetic analysis of type I polyketide synthase and nonribosomal peptide synthetase genes in Antarctic sediment. Extremophiles, 12, 97-105 (2008).

Zhao, J., N. Yang, X. Chen, Q. Jiang and R. Zeng: Phylogenetic diversity of Type I polyketide synthase genes from sediments of Ardley Island in Antarctica. Acta Oceanol. Sin., 30, 104-111 (2011).

Zhang, Z., H.X. Pan, G.L. Tang: New insights into bacterial type II polyketide biosynthesis. F1000Res., 6, 172 (2017).

Ziemert, N., M. Alanjary and T. Weber: The evolution of genome mining in microbes-a review. Nat. Prod. Rep., 33, 988-1005 (2016). 